\title{
Review of: "The natural compound TMYX reduces SAN cells rate by antagonizing the CAMP modulation of $f$-channels"
}

\author{
Eiichi Kumamoto ${ }^{1}$ \\ 1 Saga University
}

Potential competing interests: The author(s) declared that no potential competing interests exist.

Piantoni, Paina and collaborators examined the effect of a traditional Chinese medicine drug, Tongmai Yangxin (TMYX), on the electrophysiological activities of sainoatrial (SNA) myocytes isolated from rabbits by using the patch-clamp technique. As a result, they found out that TMYX dose-dependently reduces the spontaneous firing rate of the myocytes with a decreased early diastolic depolarization in a reversible manner. This TMYX action was due to a negative shift of the activation curve of funny current $\left(\mathrm{I}_{\mathrm{f}}\right)$. The TMYX activity did not involve the activation of muscarinic ACh and adenosine A1 receptors and was mediated by a reduction of CAMP-induced stimulation of a pacemaker $(\mathrm{HCN})$ channel involved in the $\mathrm{I}_{\mathrm{f}}$ through a direct antagonistic action on a CAMP-induced allosteric modulation of the channel. It was concluded that TMYX slows the spontaneous firing rate of SAN myocytes by directly antagonizing $\mathrm{I}_{\mathrm{f}}$ activation produced by CAMP. Although this finding may be interesting, there are so many points that should be addressed and be amended as follows:

Major points:

1. The first paragraph on page 5: in order to mention “TMYX and ivabradine inhibit the $\mathrm{I}_{\mathrm{f}}$ current with different molecular mechanisms", the authors should here explain what is molecular mechanism for the $I_{f}$ inhibition produced by ivabradine. Please amend this point.

2. The second paragraph of page 5 : is the suppression of firing rate by TMYX not more than $92.7 \%$ ? Is the solubility of TMYX $60 \mathrm{mg} / \mathrm{ml}$ ? Please reply to these questions.

3. Page 6: it will be better that the abbreviations of AP parameters used in Fig. 1C are given in its figure legend but not in the text. It is unnecessary to give in the text the percentages of the changes in AP parameters, as shown in Fig. 1C.

4. Figure 1: the authors should mention what is the dotted line in Fig. 1A, lower. AP waveforms in the absence and presence of TMYX should be shown in different colors. Each point in Fig. 1B represents mean but not mean \pm SEM. Please amend this point in the legend of Fig. 1. It is not clear whether " $n=68$ " in the legend shows either the total number of cells examined or the cell number of cells examined at each concentration. Please make this point clear.

5. Page 8: it is not clear how half-activation and full activation voltages were estimated to be close to -65 
$\mathrm{mV}$ and $-125 \mathrm{mV}$, respectively. Please make this point clear. In the case of " $(\mathrm{n}=6$ cells)", the authors should give the average and SEM values of the results obtained.

6. Figure 2 and this figure's legend: it will be better that "-35 mV" is put in the voltage protocol of Fig. $2 \mathrm{~A}$. "pA/pF" should be explained briefly. "-65 mV/1.5 s and - $125 \mathrm{mV} / 0.5 \mathrm{~s}$ " is an ambiguous expression; please amend this point. It is not clear what is the voltage protocol used to obtain the results shown in Fig. 2B and C; please amend this point. Please discuss what the reversal potentials obtained in Fig. 2C mean.

7. The legend of Fig. 3: the holding potential used in Fig. 3A should be stated in this legend. It is unnecessary to give in this legend individual values of percentage changes shown in Fig. 3B and D. "N.S. Not Significant" should not be repeated.

8. The second paragraph on page 11 and the first paragraph on page 12: it is unnecessary to repeat the percentage values shown in Fig. 3B and D.

9. Fig. 3C: AP waveforms in the absence and presence of drugs should be shown in different colors.

10. The second paragraph on page 12: since only DPCX effect is examined in Fig. S3, "adenosine receptor" should be replaced by "adenosine Al receptor".

11. The legend of Fig. 4: the holding potential used in Fig. 4A should be stated in this legend. In (A), "(top, $n=15)$ ", "(middle, $n=6)$ " and "(bottom, $n=6)$ " should be deleted. It is enough to give the $n$ values in (B). It is unnecessary to repeat the percentage values shown in Fig. 4B.

12. The first paragraph on page 14: it is unnecessary to repeat the percentage values shown in Fig. 4B.

13. Figure S4 and this figure's legend: the authors should give representative current traces showing the effects of IBMX, cAMP and forskolin. It is unnecessary to repeat in the legend the percentage values shown in Fig. S4.

14. The second paragraph on page 15: it is unknown from what comparison $P=0.125$ was obtained. Please make this point clear.

15. The legend of Fig. 5: " $n=4-6$ " should be written in B but not A. not "Hill fittings" but "Hill curve fittings"?

16. Figure 5B, left: the number of data points for curve fitting is too small. In particular, (CAMP+TMYX)/Cont does not attain a maximal level. Therefore, the authors should do experiments with more cAMP concentrations.

17. Figure $5 \mathrm{~B}$, right: the dotted line at $<1 \mu \mathrm{M}$ should not be drawn, because there are no data points.

18. The second paragraph on page 19: "HCN/funny channel" is used without any explanations. There is no explanation about a relation between $\mathrm{HCN} /$ funny channel and $\mathrm{I}_{\mathrm{f}}$. This relation should be stated in Introduction. Please amend these points.

19. The second paragraph on page 20: it will be better to show the Schild plot as a supplementary figure.

20. The first paragraph on page 21: "TRIP8b" should be explained in more detail.

21. The second paragraph on page 21: "cell cAMP" itself is not reduced; "cell cAMP" should be expressed in different word. The same is true for "cellular cAMP" in the first paragraph on page 20; please correct. 
Please expand "PKA".

22. The third paragraph on page 22: what is a putative substance effective in inhibiting $\mathrm{I}_{\mathrm{f}}$ among flavonoids, coumarins, iridoid, glycosides, saponins, lignans and so on, which are contained in TMYX? Please discuss about this matter.

Specific points:

1. Full title: "TMYX" and "SAN" should be expanded; "spontaneous firing" should be put before "rate"; "rabbit should be inserted in the title.

2. Abstract: "," following "(TMYX)" should be deleted; not "antagonizes" but "antagonizing"; it will be better that "muscarinic stimulation" and " $\beta$-blockers" are replaced by different words; "pacemaker channels" are somewhat ambiguous words and thus "HCN channels" should be mentioned. (2/6 $\mathrm{mg} / \mathrm{ml}),(-20.8 /-50.2 \%),(-50.1 /-76.0 \%)$ and $(-6.7 /-11.9 \mathrm{mV})$ are inaccurate expressions and thus should be expressed in different words.

3. Keywords: "TMYX" should be expanded.

4. The first paragraph on page 5 : the expression of " $I_{f}$ current" is inappropriate, because " $I_{f}$ " is used as a current, as judged from "pacemaker current $\left(I_{f}\right)$ ". Please amend this point throughout the text. "cholinergic-like" should be "acetylcholine-like". Please amend this point. " $\beta$-blocker" should be replaced by different words including "noradrenaline".

5. The second paragraph on page 5 : in ( $2 \mathrm{mg} / \mathrm{ml}$... respectively), please put ";" following " $\mathrm{n}=12$ ". Moreover, the usage of "respectively" is inappropriate. Please amend this point.

6. Pages 10 and 11: not "muscarinic blocker" but "muscarinic receptor blocker".

7. The first paragraph on page 14: not "cAMP, was" but "cAMP, were".

8. Page 16: not "Hill fittings" but "Hill curve fittings"?

9. The first paragraph on page 18: since Wenxin Keli is a mixture of various chemical substances, it is unlikely that this TCM drug binds to atrial $\mathrm{Na}^{+}$channels. Please amend this point.

10. The third paragraph on page 19: not "IK( $(A C h)$ " but "IK $(A C h)$ ". $I K_{(A C h)}$ should be explained shortly.

11. The second paragraph on page 20: not "display" but "displays"; "(I.H., 1975)" should be mentioned in detail; not "presence/absence" but "presence and absence".

12. The third paragraph on page 22: please correct "Akimoto et al. (Akimoto et al., 2018)".

13. The second paragraph on page 23: not "Tyrode's to block" but "Tyrode's solution to block".

14. The first paragraph on page 24: the location of "Sigma-Aldrich Corporation" should be mentioned. The authors should state the concentration of stock solution of the drug used and the solvent used to dissolve the drug.

15. The third paragraph on page 24: "USA" should be given.

16. The fourth paragraph on page 24: not "Action Potentials (AP)" but "Action Potentials (APs)"; “... in (Bucchi et al., 2007)." should be revised.

17. The fourth paragraph on page 25: "current amplitude" is not elicited; please amend this point. 
18. There appear to be more mistakes than pointed out above. The authors should check this manuscript very carefully. 